\title{
Gingival crevicular fluid alkaline phosphatase activity in relation to pubertal growth spurt and dental maturation: A multiple regression study
}

\author{
Perinetti, Giuseppe *; Contardo, Luca * \\ * Department of Medical, Surgical and Health Sciences, University of Trieste, Italy
}

\begin{abstract}
Introduction: The identification of the onset of the pubertal growth spurt has major clinical implications when dealing with orthodontic treatment in growing subjects.

Aim: Through multivariate methods, this study evaluated possible relationships between the gingival crevicular fluid (GCF) alkaline phosphatase (ALP) activity and pubertal growth spurt and dentition phase.

Materials and methods: One hundred healthy growing subjects ( 62 females, 38 males; mean age, $11.5 \pm 2.4$ years) were enrolled into this doubleblind, prospective, cross-sectional-design study. Phases of skeletal maturation (pre - pubertal, pubertal, post - pubertal) was assessed using the cervical vertebral maturation method. Samples of GCF for the ALP activity determination were collected at the mesial and distal sites of the mandibular central incisors. The phases of the dentition were recorded as intermediate mixed, late mixed, or permanent. A multinomial multiple logistic regression model was used to assess relationships of the enzymatic activity to growth phases and dentition phases.

Results: The GCF ALP activity was greater in the pubertal growth phase as compared to the pre - pubertal and post - pubertal growth phases. Significant adjusted odds ratios for the GCF ALP activity for the pre - pubertal and post - pubertal subjects, in relation to the pubertal group, were 0.76 and 0.84 , respectively. No significant correlations were seen for the dentition phase.

Conclusions: The GCF ALP activity is a valid candidate as a non - invasive biomarker for the identification of the pubertal growth spurt irrespective of the dentition phase.

Perinetti G, Contardo L. Gingival crevicular fluid alkaline phosphatase activity in relation to pubertal growth spurt and dental maturation: A multiple regression study. South Eur J Orthod Dentofac Res. 2016;3(1):5-10.
\end{abstract}

Submitted: December 8, 2015; Revised: February 26, 2016; Published: April 2, 2016

\section{INTRODUCTION}

The identification of the skeletal maturity, with particular regard to the onset of the pubertal growth spurt, has major clinical implications when dealing with orthodontic treatment in growing subjects, especially when skeletal disharmonies are present. ${ }^{1,2}$ The correct identification of the pre - pubertal and post-pubertal growth phases on an individual basis represents a crucial issue in orthodontic diagnosis and treatment planning. Orthopedic treatment of patients with Class III malocclusion ${ }^{3}$ and rapid maxillary expansion ${ }^{4}$ achieve maximum efficacy when

\author{
Corresponding Author: \\ Perinetti Giuseppe \\ Struttura Complessa di Clinica Odontoiatrica e Stomatologica, \\ Ospedale Maggiore, \\ Piazza Ospitale 1, 34129 Trieste, Italy \\ e-mail: G.Perinetti@fmc.units.it
}

performed at a pre - pubertal growth phase. On the contrary, in Class II subjects the amount of supplementary mandibular growth induced by functional appliances appears to be significantly greater when the treatment is performed during the pubertal growth phase. ${ }^{5,6}$ For this reason, several indicators of the skeletal maturity have been investigated over the last five decades. ${ }^{1,2,7,8}$ While chronological age showed to be an unreliable indicator of the onset of the pubertal growth spurt, ${ }^{2,7}$ other radiographical methods proved a good reliability and diagnostic performance. $1,2,7,8$

The use of biomarkers has been proposed very recently as a new aid in assessing individual skeletal maturity, with the advantage of being related to the physiology of the patient and of avoiding the use of radiations. The very scarce data reported to date include molecular constituents from the serum, such as IGF-I, ${ }^{9}$ or from the gingival crevicular fluid (GCF), such as alkaline phosphatase (ALP). ${ }^{10}$ In particular, a previous study ${ }^{10}$ on the 
relationships between skeletal maturity and GCF ALP activity was limited by the bivariate correlation analyses, while it did not consider possible confounding factors such as the dentition phase. On the contrary, multivariate analyses would better define or quantify possible relationships of the GCF ALP activity with the pubertal growth spurt. For instance, other factors such as sex or chronological age are to be taken into account, especially considering the older ages of the post - pubertal subjects as compared to the pre - pubertal subjects. Moreover, it is not defined yet whether chronological age has per se an effect on the serum ALP activity, which in turn would be responsible for the corresponding GCF activity, or whether dental eruption has an effect on the local GCF ALP activity.

Therefore, the present prospective, double-blind study was aimed at evaluating possible relationships of the GCF ALP activity with the pubertal growth spurt and dentition phase. In particular, a large sample of growing subjects was enrolled to allow proper multivariate analyses, mainly addressed to define potential of this biomarker in the identification of the onset of the pubertal growth spurt in patients scheduled for orthodontic treatment.

\section{MATERIALS AND METHODS}

\section{Study population and design}

This study enrolled 100 consecutive subjects, including 62 females and 38 males (mean age, $11.5 \pm 2.4$ years; range, 7.617.7 years), seeking orthodontic treatment and who had never been treated before. A signed informed consent was obtained from the parents of the subjects prior to entry into the study, and the protocol was reviewed and approved by the local Ethical Committee. The following enrolment criteria were observed: (i) age between 7 and 18 years; (ii) intermediate or late mixed, or early permanent phases of dentition; (iii) good general health with absence of any nutritional problems; (iv) no use of antiinflammatories or antibiotics in the month preceding entry to the study; (v) probing depth values not exceeding $4 \mathrm{~mm}$ for the whole dentition, and $3 \mathrm{~mm}$ for the anterior sextants; and (vi) full-mouth plaque score (FMPS) and full-mouth bleeding score $(\mathrm{FMBS}) \leq 25 \%$. Part of the sample herein investigated was included in previous studies. ${ }^{10,11}$

The subjects were scheduled for enrolment at their first clinical examination; subsequently, during a second visit 7 to 10 days prior to GCF collection, they underwent a session of professional supragingival and subgingival scaling, and also received repeated oral hygiene instructions. Moreover, over the days between professional scaling and GCF collection, the subjects were asked to rinse their mouths out twice a day with $0.012 \%$ chlorhexidine mouthwash, and were not allowed to take any anti-inflammatories or antibiotics. At the last clinical session, when GCF was collected for ALP activity determination, their clinical parameters were recorded, and dental panoramic radiographs and lateral cephalograms were taken immediately after GCF collection.

\section{Appraisal of individual skeletal maturity}

Appraisal of skeletal maturity was carried out through the cervical vertebral maturation (CVM) method on lateral cephalograms. This method comprises six stages (CS1-6) for cervical vertebral maturation. ${ }^{1}$ Briefly, these are defined as:

CS1: when the lower borders of the second, third and fourth vertebrae (C2, C3 and C4) are flat and the bodies of C3 and C4 are trapezoid in shape. CS1 occurs at least two years before the pubertal growth spurt.

CS2: when only the lower border of $\mathrm{C} 2$ is concave and the bodies of C3 and C4 are trapezoid. CS2 occurs one year before the growth spurt.

CS3: when the lower borders of both $\mathrm{C} 2$ and $\mathrm{C} 3$ have concavities and the bodies of $\mathrm{C} 3$ and $\mathrm{C} 4$ are either trapezoid or rectangular horizontal in shape. CS3 marks the ascending portion of the growth spurt.

CS4: when the lower borders of $\mathrm{C} 2$ to $\mathrm{C} 4$ have concavities, and the bodies of both C3 and C4 are rectangular horizontal. CS4 marks the descending portion of the growth spurt.

CS5: when the lower borders of $\mathrm{C} 2$ to $\mathrm{C} 4$ have concavities, and at least one of the bodies of C3 and C4 is square. CS5 occurs one year after the growth spurt.

CS6: when the lower borders of $\mathrm{C} 2$ to $\mathrm{C} 4$ have concavities, and at least one of the bodies of $\mathrm{C} 3$ and $\mathrm{C} 4$ is rectangular vertical. CS6 occurs at least two years after the growth spurt.

The CVM method has been preferred over other indicators of skeletal maturity because its reliability ${ }^{1,12}$ and repeatability provided that raters are well-trained. ${ }^{13}$ An experienced orthodontist who was blinded to the GCF ALP activities assessed the skeletal maturity of the subjects. Finally, the subjects were clustered into three groups according to their growth phases, as pre - pubertal (CS1 and CS2), pubertal (CS3 and CS4) and post - pubertal (CS5 and CS6).

\section{Appraisal of the dentition phase}

The appraisal of the phase of the dentition was carried out according to the following definitions: ${ }^{14}$ i) intermediate mixed dentition, when the permanent incisors and first molars had fully erupted, with presence of the deciduous canine, first and second molars; ii) late mixed dentition, when the deciduous canines and molars had exfoliated, with eruption of the permanent canines and premolars; and iii) permanent dentition, when all of the permanent teeth were present (possible presence of second molars; absence of third molars). The assessment of the phases of the dentition was performed by a single operator by intraoral evaluation, as well as on dental casts and dental panoramic radiographs when needed.

\section{Clinical monitoring and GCF collection procedures}

The intra-oral clinical examination was performed by a single operator (GP) on four sites at the mandibular central incisor 
(mesial, distal, medio-buccal and medio-palatal/lingual), as previously described. ${ }^{10}$ Briefly, the presence of supragingival plaque $(\mathrm{PL}+)$, the gingival bleeding within $15 \mathrm{sec}$. after probing $(\mathrm{BOP}+)$, and the probing depth (PD) were recorded. A single expert examiner collected the clinical data in all subjects. Contamination of the GCF was minimized by recording the PL+ before carefully cleaning the tooth with a sterile curette, then by collecting GCF and subgingival plaque from the isolated area, and finally by recording the PD and $\mathrm{BOP}+$. The GCF collection was performed at both the mesial and distal sites on each of thelower central incisors as previously described. ${ }^{10,11}$ Briefly, endodontic \#25 standardized sterile paper strips (Inline; Torino, Italy) were inserted $1 \mathrm{~mm}$ into the gingival crevice and left insitu for $60 \mathrm{sec}$. (Figure 1). These four samples were pooled, were transferred to plastic vials and immediately stored at $-80^{\circ} \mathrm{C}$, until analyzed.

Figure 1. The gingival crevicular fluid collection. Four samplings were performed at the mesial and distal aspects of each of the mandibular central incisors by using endodontic \#25 standardized sterile paper strips.

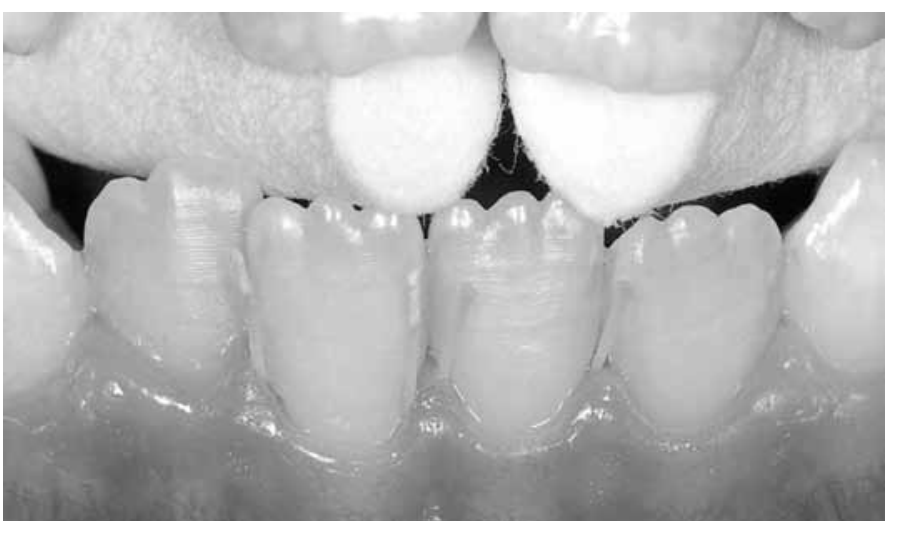

\section{Enzymatic activity determination}

The biochemical assays were performed by a single operator (GP) who was blinded to the growth phases. The four GCF samples were resuspended in $200 \mu \mathrm{l}$ buffer containing $100 \mathrm{mM}$ Tris and $20 \mathrm{mM} \mathrm{MgCl} 2(\mathrm{pH} 9.8 \pm 0.1)$ and $6 \mathrm{mM}$ p-nitrophenol phosphate. The samples were then incubated at $3{ }^{\circ} \mathrm{C}\left( \pm<0.1^{\circ} \mathrm{C}\right.$ fluctuations) for $2 \mathrm{~h}$, whereby the ALP in the samples hydrolyses the p-nitrophenyl phosphate to p-nitrophenol and inorganic phosphate. The reactions were then stopped by adding $5 \mu \mathrm{l} 3 \mathrm{M}$ $\mathrm{NaOH}$, and the rates of increase in absorbance were read with a spectrophotometer at $405 \mathrm{~nm}$. 10,15 The relevant control for each analysis consisted of the reagent and the Tris buffer without the sample. Using 18.45 as the p-nitrophenol $\mathrm{mM}$ absorptivity, the absorbance was converted into enzyme activity units ( 1 unit $=1 \mathrm{mmol}$ of p-nitrophenol released per minute at $37^{\circ} \mathrm{C}$ ) and expressed as total activity in $\mathrm{mU} /$ sample. $^{10}$

\section{Sample size calculation}

Sample size of at least 22 subjects for each of the 3 groups according to skeletal maturation (pre - pubertal, pubertal and post - pubertal) was set to detect an effect size (ES) coefficient for the GCF ALP activity of 0.8 between any two of them, with an alpha set at 0.01 and a power of $0.8 .{ }^{16}$ The ES coefficient is the ratio of the difference between the mean recordings of two different groups, i.e. pre - pubertal and pubertal groups, divided by the within - subject standard deviation (SD). An ES coefficient of 0.8 or more is regarded as to a 'large effect', ${ }^{16}$ i.e. clinically relevant correlation between the investigated parameter and the growth phase. ${ }^{17}$

\section{Data analysis}

The SPSS software, version 13.0 (SPSS ${ }^{\bullet}$ Inc., Chicago, Illinois, USA) was used to perform the statistical analyses. Parametric methods were used only for those continuous data sets that met the required assumptions of normality of the distributions and equality of variances, tested by the Shapiro-Wilk and Levene tests, respectively.

The significance of the differences in the sex distribution and chronological age among the experimental groups was tested by a chi-squared analysis, and a one-way analysis of variance (ANOVA) followed by a Bonferroni-corrected Student t-test for pairwise comparisons. Among the different groups, the significance of the differences in the $\% \mathrm{PL}+$ and $\% \mathrm{BOP}+$ was assessed by a Kruskal-Wallis test, while the significance of the difference in the mean PD was assessed by a one-way ANOVA. The chi-squared analysis assessed the significance of the differences in the distributions of the dentition phases among the different groups. Regarding the pairwise comparisons of these distributions between the different growth phases, proper Bonferroni-corrected chi-squared analysis or Fisher exact test, in case of $2 \times 2$ crosstabulations, were employed. Moreover, the Kruskal-Wallis test, followed by a Bonferroni-corrected MannWhitney U-test, assessed the significance of the differences in the enzyme activities between the different groups.

With the aim of examining the adjusted correlations between dentition phase and GCF ALP activity (explanatory variables) and the growth phase (dependent variables), a multinomial multiple logistic regression model was used. This model accounts for a multinomial depended variable, such as the phases in skeletal maturation, in which the pubertal growth phase was considered as the reference category. Dentition phase and GCF ALP activity were entered as categorical and continuous variables, respectively. The main parameter provided by this model is the odds ratio (OR), which represent the ratio of the odds of an event occurring in one group to the odds of it occurring in another group. An odds ratio of 1 indicates that the condition or event under study is equally likely to occur in the compared groups, i.e. GCF ALP activity equal between any two compared groups. An odds ratio smaller than 1 indicates that the GCF ALP activity is lower in a given group as compared to the another and vice versa. Moreover, sex and age were also entered in the model to adjust the ORs and their 95\% confidence intervals (CIs). 
Because of the lack of cases in the intermediate mixed dentition in the pubertal and post - pubertal growth phase groups (see Table 2), intermediate mixed and late mixed dentition categories were pooled together. Finally, the adjusted ORs and CIs for the GCF ALP activity were calculated on units of $10 \mathrm{mU} / \mathrm{sample}$.

Weighted kappa statistics testing the intra-examiner agreement of the CVM and dentition phase appraisal showed a value above 0.90 . A P-value less than 0.05 was used for rejection of the null hypothesis.

\section{RESULTS}

The sex distribution and age of the subjects clustered according to the growth phases are shown in Table 1 . While the distribution of the sexes was similar among the compared groups, the chronological age showed a statistically significant difference between all the three groups $(\mathrm{P}<0.001)$. The clinical parameters regarding the overall periodontal conditions of the sampling teeth are shown in Table 2. The \% $\mathrm{PL}+, \% \mathrm{BOP}+$ and mean PD were generally low without any significant differences among the groups. Moreover, the number of (mesial and distal) sampling sites PL+ were minimal, while none was BOP+ (not shown).

The dentition phase distributions among the groups are shown in Table 3. The distribution of the dentition phases was significant different among the growth phase groups $(\mathrm{P}<0.001)$. In particular, the intermediate mixed dentition was recorded only for the pre - pubertal growth phase. The late mixed and permanent dentitions were seen in all of the three growth phase groups, although more prevalent in the pre - pubertal (late mixed dentition) or in the pubertal and post - pubertal growth phases (permanent dentition). All of the differences were significant at the pairwise comparisons $(\mathrm{P}<0.001)$.

The GCF ALP activity among the groupsis shown in Figure 2. The enzymatic activity recorded for the pubertal growth phase group was about 2 times greater than those for the other two groups with a difference among the groups that was significant

Figure 2. Total GCF ALP activity in the different growth phase groups ( $n=100)$. Data presented as median values (bars represent 25 th and 75 th percentile).

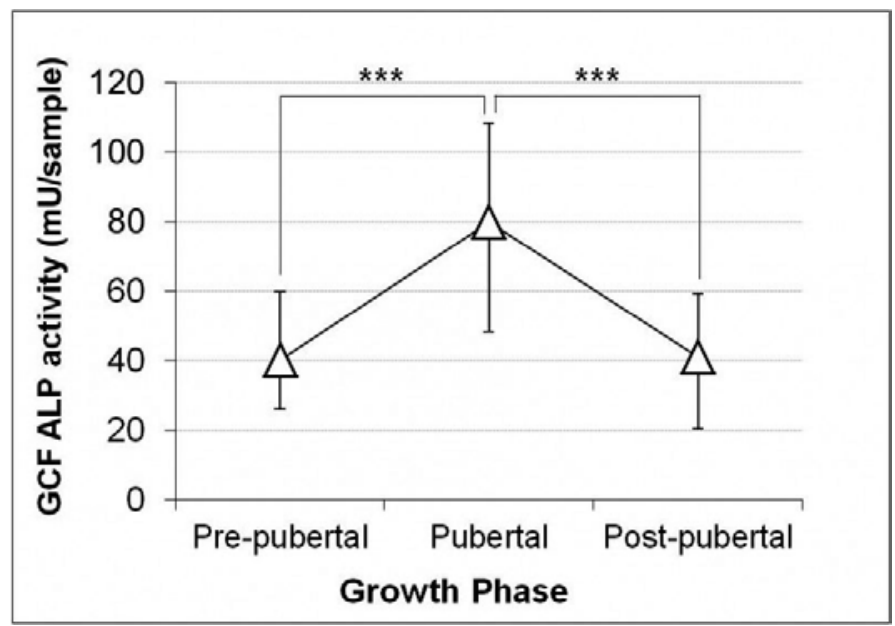

${ }^{* * *} P<0.001$.
$(\mathrm{P}<0.001)$. In particular, at the pairwise comparisons, the differences between the pubertal growth phase and the other two growth phase groups were statistically significant $(\mathrm{P}<0.001)$, while that between the pre - pubertal and post - pubertal growth phase groups was not significant.

The results of the multinomial multiple logistic regression for the adjusted correlations between GCF ALP activity and the growth phases or the dentition phasesare shown in Table 4. As compared to a mixed dentition state, a permanent dentition was negatively associated (i.e. ORs $<1$ ) with the pubertal growth phase in case of both pre - pubertal (OR, 0.27) and post pubertal (OR, 0.54) growth phases. However, the ORs were not statistically significant with noteworthy large 95\% CIs. The GCF ALP activity was negatively correlated with the pubertal growth phase in case of pre - pubertal (OR, 0.76) and post pubertal (OR, 0.84) growth phase, meaning that pre - pubertal and post - pubertal growth phase subjects had a lower GCF ALP activity as compared to subjects in the pubertal growth phase. These ORs were statistically significant $(\mathrm{P}<0.05$, at least).

\section{DISCUSSION}

The present study investigated on the relationships between the GCF ALP activity with the pubertal growth phase taking into account other possible confounding factors such as dentition phase. The adjusted ORs showed that the enzyme activity was correlated with the pubertal growth spurt at significant levels.

The different growth phase groups had a similar distribution of males and females (Table 1). On the contrary, the mean chronological ages were significantly different among the groups; nevertheless, the corresponding ranges were relatively large (Table 1), confirming that age correlates poorly with the growth phases and, in particular, with the onset of the pubertal growth spurt. ${ }^{1,2}$

As GCF composition may change during periodontal inflammation, ${ }^{18}$ tissue health is necessary to exclude any possible unwanted sources of ALP. Herein, all of the subjects received a session of professional oral hygiene, and showed optimal periodontal conditions, with very low $\% \mathrm{PL}+$ and $\% \mathrm{BOP}+$

Table 1. Sex distribution and chronological age of the subjectsin the different growth phase groups $(n=100)$.

Sex distribution is presented as number of cases and chronological age as mean $\pm S D$. Diff., significance of the differences among the groups. Note on significant differences at the pairwise comparisons: $a$, all comparisons $(P<$ 0.001). NS, not statistically significant.

\begin{tabular}{lcccc}
\hline Parameter & Pre-pubertal & $\begin{array}{c}\text { Growth phase } \\
\text { Pubertal }\end{array}$ & Post-pubertal & Diff. \\
\hline $\begin{array}{l}\text { Sex (males/ } \\
\text { females) }\end{array}$ & $18 / 29$ & $13 / 16$ & $7 / 17$ & NS \\
\hline $\begin{array}{l}\text { Chronological } \\
\text { age (years) }\end{array}$ & $9.8 \pm 1.5$ & $12.1 \pm 1.7$ & $14.1 \pm 1.9$ & P $<0.001$, a \\
\hline
\end{tabular}


scores, and with mean PD below $2 \mathrm{~mm}$, which were similar among the different growth phase groups (Table 2).

In the present study, all the subjects with an intermediate mixed dentition were in the pre - pubertal growth phase (Table 3).

Table 2. The $\% P L+$ and $\% B O P+$ and the mean $P D$ in the different growth phase groups $(n=100)$.

$\% P L+$ and $\% B O P+$ are presented as median (25th-75th percentile) and mean $P D$ as mean $\pm S D$. Diff., significance of the differences among the groups. NS, not statistically significant.

\begin{tabular}{lcccc}
\hline Parameter & \multicolumn{3}{c}{ Growth phase } & Diff. \\
\hline$\%$ Pre-pubertal & Pubertal & Post-pubertal & \\
\hline$\% \mathrm{BOP}+$ & $12.5(0-25.0)$ & $0(0-25.0)$ & $0(0-25.0)$ & NS \\
\hline Mean PD $(\mathrm{mm})$ & $12.5(0-12.5)$ & $12.5(0-18.8)$ & $0(0-12.5)$ & NS \\
\hline
\end{tabular}

Table 3. Dentition phase distributionsin the different growth phase groups $(n=100)$.

Data are presented as number of cases (percentage). Diff., significance of the differences among the groups. Note on significant differences at the pairwise comparisons: a, pre - pubertal vs. pubertal and post - pubertal $(P<0.001)$.

\begin{tabular}{lcccc}
\hline Dentition phase & \multicolumn{3}{c}{ Growth phase } & Diff. \\
& Pre-pubertal & Pubertal & Post-pubertal & \\
\cline { 1 - 4 } $\begin{array}{l}\text { Intermediate } \\
\text { mixed }\end{array}$ & $28(100 \%)$ & $0(0 \%)$ & $0(0 \%)$ & \\
\cline { 1 - 4 } Late mixed & $13(50.0 \%)$ & $9(34.6 \%)$ & $4(15.4 \%)$ & P $<0.001$, a \\
\cline { 1 - 3 } Permanent & $6(13.0 \%)$ & $20(43.5 \%)$ & $20(43.5 \%)$ & \\
\hline
\end{tabular}

These results are consistent with previous findings. ${ }^{2,7,14}$ On the contrary, subjects in the late mixed and permanent dentitions showed no clear distributions among the different growth phases (Table 3) and the adjusted ORs for the correlations between the dentition and growth phases were not significant (Table 4). In this regard, no relationship would exist between the pubertal growth spurt and the late mixed or permanent dentition phases. The dependence of tooth eruption on several environmental factors, such as loss of teeth and tooth crowding has been reported as one of the causes of the poor relationship between dentition phase and growth phases. 7,19

To date, a large number of biomarkers have been detected in the GCF, ${ }^{20}$ each responsive to a specific biological meaning, and with relevant clinical applications proposed in periodontology. ${ }^{21}$ Several molecular constituents of the GCF have also been shown to be sensitive to orthodontic tooth movement. ${ }^{22,23}$ Among these biomarkers is ALP, ${ }^{24}$ the enzyme required for bone mineralization. ${ }^{25}$ Because of the relevant role of this enzyme during growth, its serum activity increases during puberty. ${ }^{26}$ When considering GCF formation ${ }^{27}$ three potential sources can be deemed responsible for GCF ALP activity: i) serum
Table 4. Results of the multinomial multiple logistic regression for estimates of correlations of growth phase with the GCFALP activity and dentition phase $(n=100)$. $O R$, odds ratio; 95\% CI, 95\% confidence interval. ORs of 1 denote the reference category (10 mU/sample for the GCF ALP activity).

\begin{tabular}{lccccc}
\hline \multirow{2}{*}{$\begin{array}{l}\text { Explanatory } \\
\text { variable }\end{array}$} & Pubertal & \multicolumn{2}{c}{ Pre-Pubertal } & \multicolumn{2}{c}{ Post-pubertal } \\
\cline { 2 - 7 } & OR & OR & $95 \%$ CI & OR & $95 \%$ CI \\
\hline Dentition phase & 1 & 1 & & 1 & \\
\hline $\begin{array}{l}\text { Intermediate } \\
\text { and late mixed }\end{array}$ & 1 & 0,27 & $0.06-1.21$ & 0,54 & $0.10-2.98$ \\
\hline \begin{tabular}{l} 
Permanent \\
\hline $\begin{array}{l}\text { GCF ALP activity } \\
\text { (mU/sample) }\end{array}$
\end{tabular} & 1 & $0,76^{* *}$ & $0.63-0.91$ & $0,84^{*}$ & $0.71-0.99$ \\
\hline
\end{tabular}

${ }^{*} P<0.05 ;{ }^{* *} P<0.01$.

ALP (as a systemic factor); ii) maxillary/mandibular growth (as a local skeletal factor); and iii) dental eruption (as a local dentoalveolar factor). While the dependence of the GCF composition from the serum is well established ${ }^{27}$ and previous evidence indicated that local maxillary/mandibular growth has no impact on the corresponding GCF ALP activity changes, ${ }^{10}$ further evidence is necessary to establish a possible contribution from dental eruption. This process cannot be considered as bone growth, although tooth eruption can have effects on the local metabolism of the alveolar bone. If dental emergence has per se an impact of the local GCF ALP activity, the different distribution of the dentition phase among the growth phases (Table 3) have to be taken into account when analyzing possible relationships between skeletal maturity and the enzymatic activity. For this reason, multivariate models are necessary to uncover any confounding effect. Through the adjusted ORs, the present results show that the GCF ALP activity is correlated to the growth phases (Table 4); moreover, in a further regression model with the dentition phase as the dependent variable, the enzymatic activity (and the growth phase) resulted to be a nonsignificant explanatory variable (not shown). Even though the existence of a correlation between tooth emergence and local GCF ALP activity is yet to be defined, an explanation as to why dental eruption did not influence the GCF ALP activity in the present study may derive from the concept that no direct continuity between the sampling sites and eruption areas was encountered. Indeed, subjects with early mixed dentition were excluded from the study, and GCF sampling was performed only at the central incisors.

Therefore, the significantly greater GCF ALP activity of the pubertal growth phase as compared to those of the pre- and post - pubertal growth phases (Figure 2) can be explained by the serum changes in bone ALP activity during the circumpubertal growth periods. ${ }^{26}$ Although no previous studies have specifically correlated serum ALP activities with a specific indicator of skeletal maturity, the present results are consistent 
with an impact of the systemic growth on GCF composition. Of interest, the adjusted ORs for the pre - pubertal and post pubertal subjects in relation to the pubertal group were 0.76 and 0.84 , respectively (Table 4). These ORs have been calculated on the basis of a difference in $10 \mathrm{mU} / \mathrm{sample}$ of enzymatic activity as the reference level; however, the differences of the median GCF ALP activities between the pubertal subjects and the other two groups was up to about $40 \mathrm{mU} / \mathrm{sample}$ (Figure 2). The differences seen between the different growth phases would also be beyond the method error of the GCF sampling and subsequent ALP activity determination. ${ }^{28}$ This result thus supports clinical usefulness for this biomarker in assessing the pubertal growth phase, and warrants further studies on the diagnostic performance of the GCF ALP activity in assessing the pubertal growth phase.

\section{CLINICAL IMPLICATIONS AND CONCLUSIONS}

The GCF ALP activity appears to be a valid candidate as a noninvasive biomarker of the pubertal growth spurt in periodontally healthy subjects scheduled for orthodontic treatment, irrespective of the dentition phase.

The GCF analysis would also offer several advantages from a clinical standpoint. Its sampling involves a very simple, rapid and non-invasive procedure that can be performed in a clinical setting, even in the case of multiple GCF collections. Moreover, the ALP activity can be determined through routine and cheap laboratory analyses that are already available.

\section{REFERENCES}

1. Baccetti T, Franchi L, McNamara JA, Jr. The cervical vertebral maturation (CVM) method for the assessment of optimal treatment timing in dentofacial orthopedics. Semin Orthod. 2005;11:119-29.

2. Hägg U, Taranger J. Maturation indicators and the pubertal growth spurt. Am J Orthod. 1982;82(4):299-309.

3. Franchi L, Baccetti T, McNamara JA. Postpubertal assessment of treatment timing for maxillary expansion and protraction therapy followed by fixed appliances. Am J Orthod Dentofacial Orthop. 2004;126(5):555-68.

4. Baccetti T, Franchi L, Cameron CG, McNamara JA, Jr. Treatment timing for rapid maxillary expansion. Angle Orthod. 2001;71(5):34350.

5. Perinetti G, Primožič J, Franchi L, Contardo L. Treatment effects of removable functional appliances in pre - pubertal and pubertal class II patients: A systematic review and meta-analysis of controlled studies. PLoS One. 2015;28;10(10):e0141198.

6. Perinetti G, Primožič J, Furlani G, Franchi L, Contardo L. Treatment effects of fixed functional appliances alone or in combination with multibracket appliances: A systematic review and meta-analysis. Angle Orthod. 2015;85(3):480-92.

7. Bjork A, Helm S. Prediction of the age of maximum puberal growth in body height. Angle Orthod. 1967;37(2):134-43.

8. Perinetti G, Perillo L, Franchi L, Di Lenarda R, Contardo L. Maturation of the middle phalanx of the third finger and cervical vertebrae: a comparative and diagnostic agreement study. Orthod Craniofac Res. 2014;17(4):270-9.

9. Masoud M, Masoud I, Kent RL Jr., Gowharji N, Cohen LE. Assessing skeletal maturity by using blood spot insulin-like growth factor I (IGF-I) testing. Am J Orthod Dentofacial Orthop. 2008;134(2):20916.

10. Perinetti G, Baccetti T, Contardo L, Di Lenarda R. Gingival crevicular fluid alkaline phosphatase activity as a non-invasive biomarker of skeletal maturation. Orthod Craniofac Res. 2011;14(1):44-50.

11. Perinetti G, Baccetti T, Di Leonardo B, Di Lenarda R, Contardo L. Dentition phase and chronological age in relation to gingival crevicular fluid alkaline phosphatase activity in growing subjects. Prog Orthod. 2011;12(2):100-6.

12. Wong RW, Alkhal HA, Rabie AB. Use of cervical vertebral maturation to determine skeletal age. Am J Orthod Dentofacial Orthop. 2009 Oct;136(4):484.e1-6; discussion 484-5.

13. Perinetti G, Caprioglio A, Contardo L. Visual assessment of the cervical vertebral maturation stages: A study of diagnostic accuracy and repeatability. Angle Orthod. 2014;84(6):951-6.

14. Franchi L, Baccetti T, De Toffol L, Polimeni A, Cozza P. Phases of the dentition for the assessment of skeletal maturity: a diagnostic performance study. Am J Orthod Dentofacial Orthop. 2008; 133(3):395-400; quiz 476.e1-2.

15. Perinetti G, Paolantonio M, D’Attilio M, D’Archivio D, Tripodi $\mathrm{D}$, Femminella $\mathrm{B}$, et al. Alkaline phosphatase activity in gingival crevicular fluid during human orthodontic tooth movement. Am J Orthod Dentofacial Orthop. 2002;122(5):548-56.

16. Cohen J. A power primer. Psychol Bull. 1992;112(1):155-59.

17. Perinetti G, Contardo L. Posturography as a diagnostic aid in dentistry: a systematic review. J Oral Rehabil. 2009;36(12):922-36. Erratum in J Oral Rehabil. 2011;38(10):790.

18. Perinetti G, Paolantonio M, Femminella B, Serra E, Spoto G. Gingival crevicular fluid alkaline phosphatase activity reflects periodontal healing/recurrent inflammation phases in chronic periodontitis patients. J Periodontol. 2008;79(7):1200-7.

19. Grøn AM. Prediction of tooth emergence. J Dent Res. 1962;41:573-85.

20. Uitto VJ. Gingival crevice fluid--an introduction. Periodontol 2000. 2003;31:9-11.

21. McCulloch CA. Host enzymes in gingival crevicular fluid as diagnostic indicators of periodontitis. J Clin Periodontol. 1994;21(7):497-506.

22. Kavadia-Tsatala S, Kaklamanos EG, Tsalikis L. Effects of orthodontic treatment on gingival crevicular fluid flow rate and composition: clinical implications and applications. Int J Adult Orthodon Orthognath Surg. 2002;17(3):191-205.

23. Ren Y, Vissink A. Cytokines in crevicular fluid and orthodontic tooth movement. Eur J Oral Sci. 2008;116(2):89-97.

24. Ishikawa I, Cimasoni G. Alkaline phosphatase in human gingival fluid and its relation to periodontitis. Arch Oral Biol. 1970;15(12):1401-4.

25. Rodan GA. Introduction to bone biology. Bone. 1992;13 Suppl 1:S3-6.

26. Szulc P, Seeman E, Delmas PD. Biochemical measurements of bone turnover in children and adolescents. Osteoporos Int. 2000;11(4):281-94.

27. Griffiths GS. Formation, collection and significance of gingival crevice fluid. Periodontol 2000. 2003;31:32-42.

28. Perinetti G, Di Leonardo B, Di Lenarda R, Contardo L. Repeatability of gingival crevicular fluid collection and quantification, as determined through its alkaline phosphatase activity: implications for diagnostic use. J Periodontal Res. 2013;48(1):98-104. 